\title{
Cardiac output estimation using multi-beat analysis of the radial arterial blood pressure waveform: a method comparison study in patients having off-pump coronary artery bypass surgery using intermittent pulmonary artery thermodilution as the reference method
}

\author{
Bernd Saugel $^{1,2}\left(\mathbb{D} \cdot\right.$ Julia Heeschen $^{1} \cdot$ Alexander Hapfelmeier $^{3} \cdot$ Stefano Romagnoli $^{4,5} \cdot$ Gillis Greiwe $^{1}$
}

Received: 18 April 2019 / Accepted: 5 August 2019 / Published online: 27 August 2019

(C) Springer Nature B.V. 2019

\begin{abstract}
Pulse wave analysis enables stroke volume to be estimated from an arterial blood pressure waveform. Multi-beat analysis is a novel pulse wave analysis method. We aimed to investigate cardiac output (CO) estimations using multi-beat analysis of the radial arterial blood pressure waveform in patients undergoing off-pump coronary artery bypass surgery (OPCAB) using intermittent pulmonary artery thermodilution (PATD) as the reference method. This was a prospective clinical method comparison study. In 58 patients, we measured CO using PATD (PATD-CO; reference method) and simultaneously recorded the radial arterial blood pressure waveform that we used for off-line estimation of CO based on multi-beat analysis (MBACO; test method) using the Argos CO monitor (Retia Medical; Valhalla, NY, USA). The final analysis was performed using 572 paired CO measurements. We performed Bland-Altman analysis accounting for multiple observations per patient. To describe the ability of the test method to track changes in $\mathrm{CO}$ over time we computed four-quadrant plots using a central exclusion zone of $15 \%$ and calculated the concordance rate. Mean PATD-CO was $4.13 \pm 1.26 \mathrm{~L} / \mathrm{min}$ and mean MBA-CO was $4.31 \pm 1.25 \mathrm{~L} / \mathrm{min}$. The mean of the differences between PATD-CO and MBA-CO was $-0.20 \mathrm{~L} / \mathrm{min}$ with a standard deviation of $\pm 1.14 \mathrm{~L} / \mathrm{min}$ and $95 \%$ limits of agreement of -2.48 to $+2.08 \mathrm{~L} / \mathrm{min}$. The concordance rate for $\mathrm{CO}$ changes between PATD-CO and MBA-CO was $89 \%$. CO estimations using multi-beat analysis (Argos monitor) show reasonable agreement and trending ability compared with PATD-CO as the reference method in adult patients during OPCAB.
\end{abstract}

Keywords Pulse wave analysis $\cdot$ Pulse contour analysis $\cdot$ Pulmonary artery catheter $\cdot$ Swan-Ganz catheter $\cdot$ Hemodynamic monitoring $\cdot$ Hemodynamics $\cdot$ Cardiovascular dynamics $\cdot$ Anesthesia

Bernd Saugel and Julia Heeschen contributed equally to the study.

Bernd Saugel

bernd.saugel@gmx.de; b.saugel@uke.de

1 Department of Anesthesiology, Center of Anesthesiology and Intensive Care Medicine, University Medical Center Hamburg-Eppendorf, Martinistrasse 52, 20246 Hamburg, Germany

2 Outcomes Research Consortium, Cleveland, OH, USA

3 Institute of Medical Informatics, Statistics and Epidemiology, Klinikum rechts der Isar, Technische Universität München, Munich, Germany

4 Section of Anesthesia and Critical Care, Department of Health Science, University of Florence, Florence, Italy

5 Department of Anesthesia and Critical Care, AOU Careggi, Florence, Italy

\section{Introduction}

Cardiac output (CO) monitoring is recommended in highrisk surgical and critically ill patients [1-3]. Various methods for $\mathrm{CO}$ estimation with different physical measurement principles and degrees of invasiveness are available [4,5]. Uncalibrated pulse wave analysis, the estimation of stroke volume based on the analysis of the arterial blood pressure waveform, constitutes a minimally invasive method and enables $\mathrm{CO}$ to be estimated continuously [3, 6-8].

One method to mathematically analyze the radial arterial blood pressure waveform to estimate stroke volume and $\mathrm{CO}$ is the so-called multi-beat analysis (MBA) $[9,10]$. This method assumes a Windkessel model of the cardiovascular system. When estimating the Windkessel model parameters 
it attenuates the confounding effects of peripheral pulse wave reflections due to changes in the peripheral vascular resistance. The method works by reverse-engineering the arterial tree impulse response by analyzing the arterial blood pressure waveform over time scales greater than a cardiac cycle (i.e., over multiple heart beats) in order to extract a waveform that would be present in the absence of high frequency wave reflections $[9,10]$. An arterial compliance scale factor is then applied according to a proprietary formula that includes the measured blood pressure and patient age, height, weight, and sex. Thus, $\mathrm{CO}$ is estimated over several cardiac cycles and stroke volume is calculated knowing the heart rate. No external calibration is needed.

Measurements of $\mathrm{CO}$ using multi-beat analysis have been prospectively compared with aortic flow probe-derived $\mathrm{CO}$ measurements in swine [10] and retrospectively with thermodilution $\mathrm{CO}$ measurements in hemodynamically stable intensive care unit patients (using arterial blood pressure waveforms from the freely accessible Multiparameter Intelligent Monitoring in Intensive Care (MIMIC II) database) $[9,11,12]$. Just recently, a commercially available monitor using MBA for $\mathrm{CO}$ estimation from a radial arterial blood pressure signal in adults-the Argos CO monitor (Retia Medical; Valhalla, NY, USA)—became available and was cleared by the US Food and Drug Administration (FDA). The device estimates and displays CO every $5 \mathrm{~s}$ using multibeat analysis over $20 \mathrm{~s}$ or $5 \mathrm{~min}$.

To the best of our knowledge, there are no clinical data on $\mathrm{CO}$ measurements using multi-beat analysis in patients having cardiac surgery who often show rapid changes in cardiovascular dynamics. We, therefore, investigated CO estimations using multi-beat analysis of the radial arterial blood pressure waveform in a clinical method comparison study in patients having off-pump coronary artery bypass surgery $(\mathrm{OPCAB})$ using intermittent pulmonary artery thermodilution (PATD) as the reference method.

\section{Methods}

\subsection{Study design and setting}

The study was reviewed and approved by the local ethics committee (Ethikkommission der Ärztekammer Hamburg, Hamburg, Germany) and all patients gave written informed consent to participate in the study. For this clinical method comparison study we prospectively included patients scheduled for OPCAB in the University Heart Center Hamburg of the University Medical Center Hamburg-Eppendorf (Hamburg, Germany). The study was performed between May 2016 and January 2017. During surgery, we performed intermittent PATD measurements to assess reference $\mathrm{CO}$ values and simultaneously recorded the radial arterial blood pressure waveform that we used for off-line estimation of CO by MBA using the Argos CO monitor (test method). All patients were mechanically ventilated in a pressure-controlled mode with a tidal volume of $6-8 \mathrm{~mL} / \mathrm{kg}$ predicted bodyweight to maintain normocapnia.

\subsection{Inclusion and exclusion criteria}

Adult patients scheduled for OPCAB in whom continuous invasive arterial blood pressure monitoring using a radial arterial catheter and advanced hemodynamic monitoring using a pulmonary artery catheter were planned for clinical indications were eligible for study inclusion. Exclusion criteria were age $<18$ years, atrial fibrillation or other high-grade cardiac arrhythmias or missing informed consent.

\subsection{Study measurements}

During surgery, we systematically performed two sets of study $\mathrm{CO}$ measurements at six different time points (resulting in a total of 12 study $\mathrm{CO}$ measurements). One study CO measurement consisted of intermittent PATD to assess PATD-CO (reference method) and simultaneous recording of the radial arterial blood pressure waveform that we later used to estimate $\mathrm{CO}$ using MBA (MBA-CO; test method). In some patients, we were not able to perform all six planned interventions because of a shorter than expected duration of surgery: We performed measurements around six interventions in 22 patients, five interventions in 20 patients, four interventions in 7 patients, three interventions in 8 patients, and two interventions in 1 patient. The time points for study $\mathrm{CO}$ measurements were not pre-defined but determined by clinical interventions expected to markedly change the patient's cardiovascular dynamics (e.g., administration of fluid and vasopressive or inotropic medication). We performed one set of study $\mathrm{CO}$ measurements prior to the clinical intervention and one shortly after the intervention.

Intermittent PATD was performed via an ARROW pulmonary artery catheter (Teleflex Medical Europe; Co Westmeath, Ireland). For PATD-CO measurements we performed four consecutive injections of the thermal indicator $(10 \mathrm{~mL}$ ice-cold sodium chloride) randomly over the respiratory cycle and averaged the $\mathrm{CO}$ values resulting in an averaged PATD-CO.

The Argos CO monitor uses multi-beat analysis of the arterial blood pressure waveform to estimate $\mathrm{CO}$ and was the test method in this study. During PATD-CO measurements, we simultaneously recorded the arterial blood pressure waveform at a sampling rate of $100 \mathrm{~Hz}$ from the radial arterial catheter using a personal computer with dedicated data acquisition software (Dräger Data Grabber; Dräger, Lübeck, Germany). Since a good quality of the arterial blood pressure waveform is a prerequisite for pulse wave analysis 
we carefully leveled the pressure transducers to the height of the right atrium and zeroed the system to atmospheric pressure. In addition, we confirmed adequate damping properties of the tubing/transducer-system by square wave tests. In an off-line analysis, these blood pressure waveform segments (starting $20 \mathrm{~s}$ before the first and stopping $20 \mathrm{~s}$ after the last PATD measurement) were retrospectively fed into the Argos CO monitor along with patient age, sex, height, and weight. The Argos CO monitor accepts an analog arterial blood pressure waveform as an input (scaled to $100 \mathrm{mmHg} / \mathrm{V}$ ). The recorded $\mathrm{BP}$ waveform was fed into the Argos $\mathrm{CO}$ monitor via a $3.5 \mathrm{~mm}$ stereo cable, using an MP150 digital-to-analog converter (BIOPAC System, Inc., Goleta, CA, USA). In this study, we used the Argos CO monitor in the 20-s mode.

\subsection{Data processing}

We performed a total of 665 study $\mathrm{CO}$ measurements in 60 patients (convenience sampling according to availability of investigators). We excluded 47 study $\mathrm{CO}$ measurements due to the unavailability of BP waveform segments matching the corresponding PATD-CO measurements. We further excluded one segment due to unstable PATD-CO measurements. We further excluded five segments due to instability and two segments due to artifact in the BP waveform. We were interested in paired measurements (before and after interventions) and therefore excluded 38 unpaired data segments. After this process of data exclusion, in 2 patients, no study CO measurement data remained. Final analyses were performed on the remaining 572 paired study $\mathrm{CO}$ measurements from 58 patients.

\subsection{Statistical analysis}

For statistical analyses we used $\mathrm{R}$ version 3.5.0 (The $\mathrm{R}$ Foundation for Statistical Computing, Vienna, Austria). Demographic, biometric, and clinical data are presented as absolute and relative frequencies (percentages) or mean \pm standard deviation (SD). The mean $\mathrm{CO}$ values $\pm \mathrm{SD}$ were separately calculated for PATD-CO and MBA-CO.

The correlation between PATD-CO and MBA-CO was computed within subjects taking repeated observations into account and removing the between subject variability (as required for method comparison studies) [13].

To assess the agreement between PATD-CO and MBA$\mathrm{CO}$ we performed Bland-Altman analysis accounting for multiple observations per patient [14] and calculated the mean and SD of the differences, and the $95 \%$ limits of agreement $(=$ mean difference $\pm 1.96 \times \mathrm{SD}$ of the difference), and the percentage error [15]. To describe the ability of the multi-beat analysis method to track changes in $\mathrm{CO}$
Table 1 Baseline patient characteristics

\begin{tabular}{ll}
\hline Number of included patients, $\mathrm{n}$ & 58 \\
Age (years) & $70 \pm 10$ \\
Sex, female [n (\%)] & $10(17)$ \\
Height $(\mathrm{m})$ & $1.74 \pm 0.09$ \\
Weight $(\mathrm{kg})$ & $81 \pm 15$ \\
BMI $\left(\mathrm{kg} \mathrm{m}^{-2}\right)$ & $26.8 \pm 4.2$ \\
\hline
\end{tabular}

Data are displayed as absolute and relative frequencies or mean and standard deviation

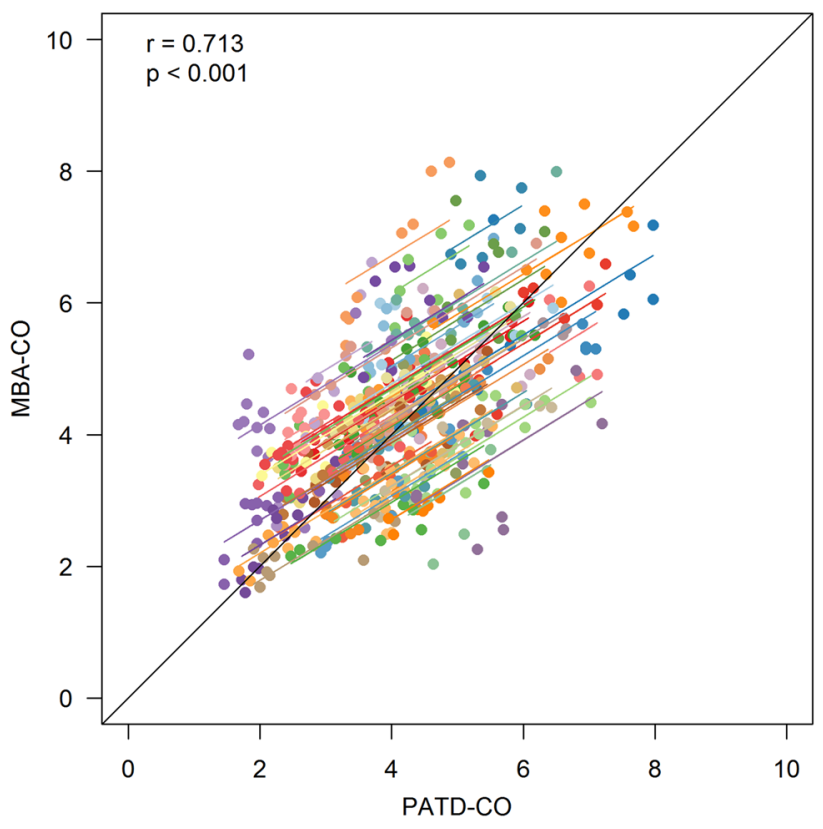

Fig. 1 Scatter plot illustrating the relation of cardiac output estimations obtained with multi-beat analysis (MBA-CO; test method) and cardiac output measurements with pulmonary artery thermodilution (PATD-CO; reference method). The within-subject correlation coefficient ( $r$ ) is presented along a respective illustration of the intra-individual linear trend. Patients are distinguished by color

over time we computed four-quadrant plots showing relative $\mathrm{CO}$ changes using a central exclusion zone of $15 \%$ and calculated the concordance rate $[16,17]$.

\section{Results}

The demographic, biometric, and clinical data of the patients are shown in Table 1. Mean PATD-CO was $4.13 \pm 1.26 \mathrm{~L} /$ min and mean MBA-CO was $4.31 \pm 1.25 \mathrm{~L} / \mathrm{min}$. In Fig. 1, the relation and within-subject correlation of PATD-CO and of MBA-CO are shown. The mean of the differences between PATD-CO and MBA-CO was $-0.20 \mathrm{~L} / \mathrm{min}$ with a $\mathrm{SD}$ of $\pm 1.14 \mathrm{~L} / \mathrm{min}$ and $95 \%$ limits of agreement of -2.48 to $+2.08 \mathrm{~L} / \mathrm{min}$ (Fig. 2). The percentage error was $50.8 \%$. 


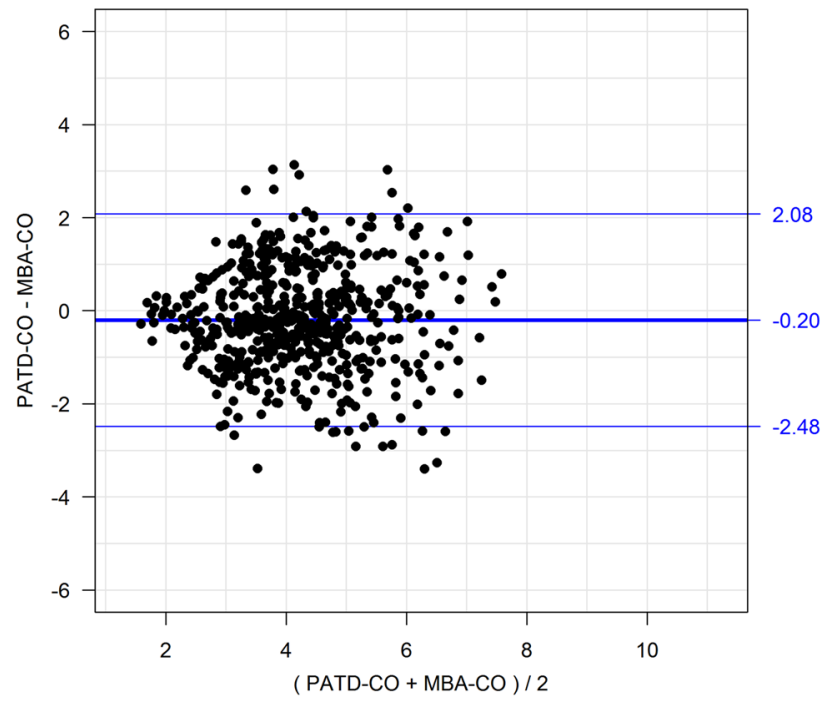

Fig. 2 Bland-Altman plot showing the agreement between cardiac output estimations obtained with multi-beat analysis (MBA-CO; test method) and cardiac output measurements with pulmonary artery thermodilution (PATD-CO; reference method). The mean of the differences between MBA-CO and PATD-CO (bold horizontal line) and the upper and lower 95\% limits of agreement (thin horizontal lines) are shown

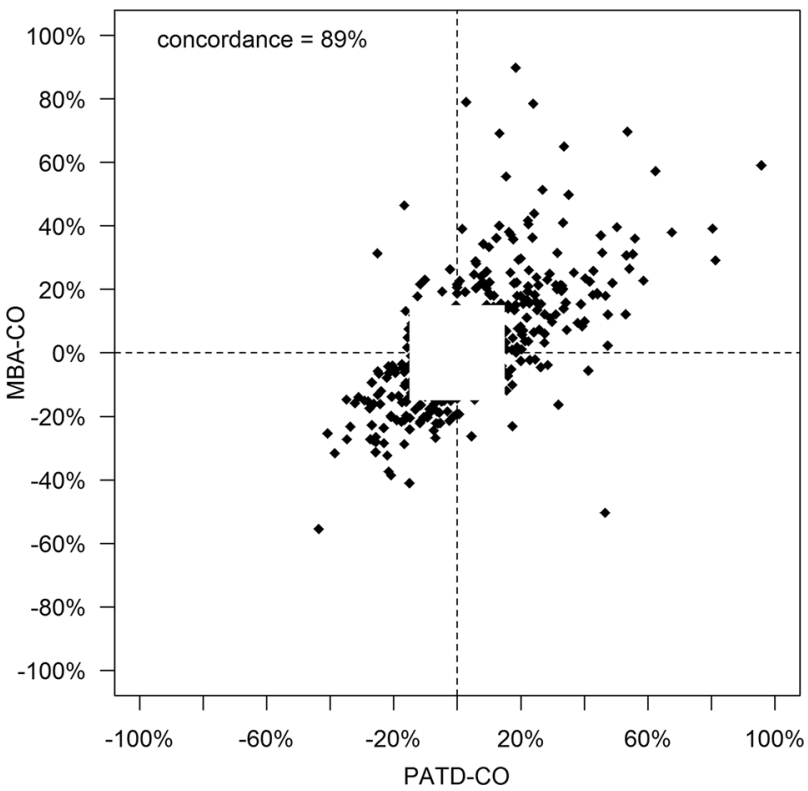

Fig. 3 The capability of the multi-beat analysis method to track relative changes in cardiac output is shown in a four-quadrant plot with an exclusion zone of 15\%. MBA-CO, cardiac output estimations obtained with multi-beat analysis (test method); PATD-CO, cardiac output measurements with pulmonary artery thermodilution (reference method)

The ability of MBA-CO to track changes in PATD-CO is illustrated in Fig. 3. The concordance rate for $\mathrm{CO}$ changes between PATD-CO and MBA-CO was $89 \%$.

\section{Discussion}

This was a method comparison study investigating $\mathrm{CO}$ estimations using multi-beat analysis of the radial arterial blood pressure waveform (Argos monitor) in patients having OPCAB using intermittent PATD as the reference method. $\mathrm{CO}$ estimations using multi-beat analysis showed reasonable agreement and trending ability compared with PATD-CO.

The arterial system is a branching tree of elastic arteries; therefore, the mathematical analysis of the arterial blood pressure waveform is complex because various cardiovascular factors-including the contractility of the heart, vascular compliance, aortic impedance, and peripheral vascular resistance-influence blood flow wave propagation and reflection and thus the shape of the waveform [18]. Different mathematical models have been proposed to describe the blood flow through the arterial system [18, 19]. "Windkessel models" explain the shape of the arterial blood pressure waveform by the interactions between the blood flow (stroke volume) and the arterial compliance (i.e., the elastic properties and the buffering capacity of the aorta and the large arteries) and the peripheral resistance generated by the small arteries and arterioles [6,18-21]. The addition of other parameters, the characteristic aortic impedance [22] and the inertia of the blood [23,24], results in the more complex three-element and four-element Windkessel models $[18,19,22]$.

The analysis of the arterial blood pressure waveform, referred to as pulse wave analysis, can be used to continuously estimate stroke volume and $\mathrm{CO}$ [3, 6-8]; various different methods and mathematical algorithms have been proposed for pulse wave analysis [7, 25, 26]. Assuming a linear relationship between pulse pressure and stroke volume and deriving arterial compliance from biometric factors and waveform characteristics, pulse pressure can be used as a surrogate for stroke volume $[6,26]$. Other methods use the systolic part of the area under the arterial blood pressure curve to estimate stroke volume or perform a so-called "pulse power analysis" by converting the arterial blood pressure waveform into a sine wave and analyzing it using the root mean square method [6, 26]. However, because of wave phenomena and transmission effects within the arterial tree the shape of an individual arterial blood pressure waveform is not solely a function of stroke volume, but a complex function of cardiac ejection, wave propagation, and wave reflections.

The MBA method investigated in our study analyzes the waveform over longer time scales that include multiple heart beats, estimates the contribution of the different heart beats within this sequence, attenuates the confounding effects of complex wave reflections, and estimates a theoretical arterial blood pressure waveform that would be generated by one single isolated heart beat $[9,10,27]$. 
The analysis of this theoretical arterial blood pressure waveform enables peripheral vascular resistance to be estimated (assuming that arterial compliance is constant) and $\mathrm{CO}$ to be calculated using mean arterial pressure and peripheral vascular resistance $[9,10,27]$.

In our study, $\mathrm{CO}$ estimations using multi-beat analysis (Argos monitor) showed reasonable agreement and trending ability compared with PATD-CO as the reference method in adult patients during OPCAB. Pulse wave analysis might especially be useful to indicate changes in $\mathrm{CO}$. We revealed a concordance rate between changes in PATD-CO and changes in MBA-CO of $89 \%$. In comparison to our study, the concordance rate was lower in two recent studies investigating the trending capabilities of an established pulse wave analysis algorithm (FloTrac; Edwards Lifesciences, Irvine; CA, USA) compared with PATD in patients having cardiac surgery using cardiopulmonary bypass $[28,29]$.

We solely included patients having OPCAB. We chose this patient collective for several reasons. First, patients having OPCAB in our institution are routinely monitored with a radial arterial catheter and a pulmonary artery catheter. This allows performing studies on pulse wave analysis in comparison with the clinical gold standard method (i.e., intermittent PATD) [4, 30]. Further, patients having OPCAB are highly complex with regard to alterations and changes in cardiovascular dynamics. Rapid changes in the hemodynamic status of the patients during OPCAB made it possible to assess the ability of multibeat analysis to track rapid changes in $\mathrm{CO}$. However, on the other hand, marked alterations and rapid changes in vasomotor tone are a well-described limitation of pulse wave analysis methods and may have influenced the measurement performance of multi-beat analysis in our study [6-8]. In addition, patients with coronary artery disease may have altered arterial compliance. Therefore, our results should not indiscriminately be transferred to other groups of surgical or critically ill patients, especially patients who are hemodynamically more stable. In addition, this was a single center study performed in a tertiary care medical university center. Therefore, our results might also not be generalizable to other clinical settings. Further, the blood pressure waveform segments were retrospectively fed into the Argos $\mathrm{CO}$ monitor for $\mathrm{CO}$ estimation (off-line analysis). Thus, further studies using the Argos $\mathrm{CO}$ monitor at the bedside in less complex patients and other clinical settings are needed.

\section{Conclusions}

CO estimations using multi-beat analysis (Argos monitor) show reasonable agreement and trending ability compared with PATD-CO as the reference method in adult patients during OPCAB.

Acknowledgements We thank Ricardo Toledo-Crow, PhD (Advanced Science Research Center, City University of New York, New York, NY, USA) for his support with off-line analysis, i.e., feeding arterial blood pressure waveforms into the Argos CO monitor (Retia Medical) for $\mathrm{CO}$ estimation.

Funding The study was funded with an institutional restricted research grant by Retia Medical (Valhalla, NY, USA).

\section{Compliance with ethical standards}

Conflicts of interest BS has received institutional restricted research grants from Retia Medical (Valhalla, NY, USA). BS collaborates with Pulsion Medical Systems (Feldkirchen, Germany) as a member of the medical advisory board and has received institutional restricted research grants, honoraria for giving lectures, and refunds of travel expenses from Pulsion Medical Systems. BS has received research support and honoraria for giving lectures from Edwards Lifesciences (Irvine, CA, USA). BS has received institutional restricted research grants, honoraria for giving lectures, and refunds of travel expenses from CNSystems Medizintechnik (Graz, Austria). BS has received institutional restricted research grants, honoraria for consulting, and refunds of travel expenses from Tensys Medical (San Diego, CA, USA). BS has received honoraria for giving lectures from Philips Medizin Systeme Böblingen (Böblingen, Germany). SR received honoraria for giving lectures from Baxter (Rome, Italy), BBraun (Melsungen, Germany), Orion Pharma (Espoo, Finland), Medigas (Assago, Italy), and Vygon (Écouen, France). SR has received honoraria for consulting from Vygon and Medtronic (Minneapolis, MN, USA). SR has received institutional restricted research grants from Pall International (Milano, Italy) and Baxter (Rome, Italy). GG has received travel expenses from Vygon. For all other authors there is no conflict of interest to declare.

Informed consent Informed consent was obtained from all individual participants included in the study.

Research involving human participants and/or animals This method comparison study was reviewed and approved by the ethics committee (Ethikkomission der Ärztekammer Hamburg, Hamburg, Germany). All procedures performed in studies involving human participants were in accordance with the ethical standards of the institutional and/or national research committee and with the 1964 Helsinki declaration and its later amendments or comparable ethical standards. 


\section{References}

1. Vincent JL, Pelosi P, Pearse R, Payen D, Perel A, Hoeft A, Romagnoli S, Ranieri VM, Ichai C, Forget P, Della Rocca G, Rhodes A. Perioperative cardiovascular monitoring of high-risk patients: a consensus of 12 . Crit Care. 2015;19:224. https://doi. org/10.1186/s13054-015-0932-7.

2. Vincent JL, Rhodes A, Perel A, Martin GS, Della Rocca G, Vallet B, Pinsky MR, Hofer CK, Teboul JL, de Boode WP, Scolletta S, Vieillard-Baron A, De Backer D, Walley KR, Maggiorini M, Singer M. Clinical review: update on hemodynamic monitoring-a consensus of 16. Crit Care. 2011;15:229. https://doi.org/10.1186/ cc10291.

3. Saugel B, Vincent JL. Cardiac output monitoring: how to choose the optimal method for the individual patient. Curr Opin Crit Care. 2018;24:165-72. https://doi.org/10.1097/mcc.0000000000 000492.

4. De Backer D, Bakker J, Cecconi M, Hajjar L, Liu DW, Lobo S, Monnet X, Morelli A, Myatra SN, Perel A, Pinsky MR, Saugel B, Teboul JL, Vieillard-Baron A, Vincent JL. Alternatives to the Swan-Ganz catheter. Intensive Care Med. 2018;44:730-41. https ://doi.org/10.1007/s00134-018-5187-8.

5. Teboul JL, Saugel B, Cecconi M, De Backer D, Hofer CK, Monnet X, Perel A, Pinsky MR, Reuter DA, Rhodes A, Squara P, Vincent JL, Scheeren TW. Less invasive hemodynamic monitoring in critically ill patients. Intensive Care Med. 2016;42:1350-9. https://doi. org/10.1007/s00134-016-4375-7.

6. Thiele RH, Durieux ME. Arterial waveform analysis for the anesthesiologist: past, present, and future concepts. Anesth Analg. 2011;113:766-76. https://doi.org/10.1213/ANE.0b013e3182 2773ec.

7. Esper SA, Pinsky MR. Arterial waveform analysis. Best Pract Res Clin Anaesthesiol. 2014;28(4):363-80. https://doi.org/10.1016/j. bpa.2014.08.0027.

8. Jozwiak M, Monnet X, Teboul JL. Pressure waveform analysis. Anesth Analg. 2017;126(6):1930-3. https://doi.org/10.1213/ ane.0000000000002527.

9. Lu Z, Mukkamala R. Continuous cardiac output monitoring in humans by invasive and noninvasive peripheral blood pressure waveform analysis. J Appl Physiol. 2006;101(2):598-608. https ://doi.org/10.1152/japplphysiol.01488.2005.

10. Mukkamala R, Reisner AT, Hojman HM, Mark RG, Cohen RJ. Continuous cardiac output monitoring by peripheral blood pressure waveform analysis. IEEE Trans Biomed Eng. 2006;53(3):459-67. https://doi.org/10.1109/tbme.2005.869780.

11. Mukkamala R, Kuiper J, Ahmad S, Lu Z. Cardiac output monitoring in intensive care patients by radial artery pressure waveform analysis. In: Proceedings of the 26th annual international conference of the IEEE EMBS, vol. 5, pp. 3712-3715; 2004. https://doi. org/10.1109/iembs.2004.1404042.

12. Zhang G, Mukkamala R. Continuous and minimally invasive cardiac output monitoring by long time interval analysis of a radial arterial pressure waveform: assessment using a large, public intensive care unit patient database. Br J Anaesth. 2012;109(3):339-44. https://doi.org/10.1093/bja/aes099.

13. Bland JM, Altman DG. Calculating correlation coefficients with repeated observations: part 1 -correlation within subjects. BMJ. 1995;310:446.

14. Bland JM, Altman DG. Agreement between methods of measurement with multiple observations per individual. J Biopharm Stat. 2007;17(4):571-82. https://doi.org/10.1080/105434007013294 229.

15. Critchley LA, Critchley JA. A meta-analysis of studies using bias and precision statistics to compare cardiac output measurement techniques. J Clin Monit Cumput. 1999;15(2):85-91.
16. Saugel B, Grothe O, Wagner JY. Tracking changes in cardiac output: statistical considerations on the 4-quadrant plot and the polar plot methodology. Anesth Analg. 2015;121(2):514-24. https://doi. org/10.1213/ane.0000000000000725.

17. Montenij LJ, Buhre WF, Jansen JR, Kruitwagen CL, de Waal EE. Methodology of method comparison studies evaluating the validity of cardiac output monitors: a stepwise approach and checklist. Br J Anaesth. 2016;116(6):750-8. https://doi.org/10.1093/bja/ aew094.

18. Segers P, Rietzschel ER, De Buyzere ML, Stergiopulos N, Westerhof N, Van Bortel LM, Gillebert T, Verdonck PR. Three- and four-element Windkessel models: assessment of their fitting performance in a large cohort of healthy middle-aged individuals. Proc Inst Mech Eng Part H J Eng Med. 2008;222(4):417-28. https ://doi.org/10.1243/09544119jeim287.

19. Westerhof N, Lankhaar JW, Westerhof BE. The arterial Windkessel. Med Biol Eng Comput. 2009;47:131. https://doi.org/10.1007/ s11517-008-0359-2.

20. Frank O. The basic shape of the arterial pulse. First treatise: mathematical analysis. 1899. J Mol Cell Cardiol. 1990;22(3):255-77.

21. Parker KH. A brief history of arterial wave mechanics. Med Biol Eng Comput. 2009;47(2):111-8. https://doi.org/10.1007/s1151 7-009-0440-5.

22. Westerhof N, Elzinga G, Sipkema P. An artificial arterial system for pumping hearts. J Appl Physiol. 1971;31(5):776-81.

23. Burattini R, Gnudi G. Computer identification of models for the arterial tree input impedance: comparison between two new simple models and first experimental results. Med Biol Eng Comput. 1982;20(2):134-44.

24. Stergiopulos N, Westerhof BE, Westerhof N. Total arterial inertance as the fourth element of the windkessel model. Am J Physiol. 1999;276(1 Pt 2):H81-8.

25. Sun JX, Reisner AT, Saeed M, Heldt T, Mark RG. The cardiac output from blood pressure algorithms trial. Crit Care Med. 2009;37(1):72-80. https://doi.org/10.1097/CCM.0b013e3181 930174.

26. Sangkum L, Liu GL, Yu L, Yan H, Kaye AD, Liu H. Minimally invasive or noninvasive cardiac output measurement: an update. J Anesth. 2016;30(3):461-80. https://doi.org/10.1007/s0054 0-016-2154-9.

27. Reisner AT, Xu D, Ryan KL, Convertino VA, Rickards CA, Mukkamala R. Monitoring non-invasive cardiac output and stroke volume during experimental human hypovolaemia and resuscitation. Br J Anaesth. 2011;106(1):23-30. https://doi.org/10.1093/bja/ aeq295.

28. Lin SY, Chou AH, Tsai YF, Chang SW, Yang MW, Ting PC, Chen CY. Evaluation of the use of the fourth version FloTrac system in cardiac output measurement before and after cardiopulmonary bypass. J Clin Monit Comput. 2018;32(5):807-15. https://doi. org/10.1007/s10877-017-0071-6.

29. Kusaka Y, Ohchi F, Minami T. Evaluation of the fourth-generation FloTrac/Vigileo system in comparison with the intermittent bolus thermodilution method in patients undergoing cardiac surgery. J Cardiothorac Vasc Anesth. 2019;33(4):953-60. https://doi. org/10.1053/j.jvca.2018.06.017.

30. Rajaram SS, Desai NK, Kalra A, Gajera M, Cavanaugh SK, Brampton W, Young D, Harvey S, Rowan K. Pulmonary artery catheters for adult patients in intensive care. Cochrane Database Syst Rev. 2013;2:CD003408. https://doi.org/10.1002/14651858. CD003408.pub3.

Publisher's Note Springer Nature remains neutral with regard to jurisdictional claims in published maps and institutional affiliations. 